\title{
Interactive comment on "Geospatial input data for the PALM model system 6.0: model requirements, data sources, and processing" by Wieke Heldens et al.
}

\section{Anonymous Referee \#1}

Received and published: 18 May 2020

General Summary and Comments This manuscript describes a semi-automated system for preparing a set of environmental input data for the PALM model system. The PALM model is designed to simulate micro- and mesoscale flow dynamics in urban environments. Examples of the system for deriving parameter sets for three German cities are provided. The input parameters (building, soil, water, road, and vegetation properties) appear to be thoroughly defined, extensive, and complete. Overall, the manuscript is well-written, clearly presented, and recognizes previous work in this area. Although, please see the Technical Comments below. The main aspects of the manuscript that require improvement are related to the need to provide a more complete description of the PALM model to provide context for the rest of the paper, to

Printer-friendly version

Discussion paper 
include some examples of input parameters in individual sections, and the lack of any discussion regarding whether the model has been run/evaluated/validated with these parameter sets (see Specific Comments below for more details).

\section{Specific Comments}

1. Section 1, Lines 7-15: I think this paragraph should be expanded to provide more information on what PALM is and how it is structured and operates (for instance, what spatial resolution does the model typically operate at?), including some background on how it has been applied in past studies, with references. In addition, what capabilities does the model have (i.e., later in the paper there is reference to "embedded chemistry model" and "embedded multi-agent system for urban residents" and "nested simulation setup" and "compute the energy demand of single buildings". This information only slowly becomes apparent as one reads through the paper. Maybe there is an existing graphic from a previous paper that could help the reader visualize the environment that the model is attempting to represent (e.g., what types of surfaces are represented?). This would be valuable to provide some context for the rest of the paper describing the input data. This may be particularly important since the Maronga et al. 2019 paper that apparently describes PALM is listed as submitted? 2. Section 2: It would be useful and clarifying to provide one or two concrete examples for each of the subsections describing the input requirements, so that the reader doesn't necessarily have to go to the tables to understand each section (particularly since these tables are in the appendix). For instance, what are some of the water body types and parameters described in section 2.6.3, what are some of the soil types and parameters described in section 2.7, etc. 3. I didn't see any indication that the model has actually been run with this set of data for the three example cities? Was it run and the output verified/validated? What were the problems encountered and what were the overall results?

Printer-friendly version

Discussion paper

1. Abstract: Is PALM an acronym? I don't see it defined in the abstract. 2. Abstract,

\section{Technical Comments}

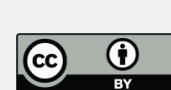


Line 5: Suggest rewording or removing "standardized, hereafter called PALM input data standard". Maybe "...follows a standardized format, hereafter called the PALM input data standard". On the other hand it may not be necessary to call this out in the abstract. Later in the paper this is referred to as "the so-called PALM input data standard". 3. Page 2, Line 4: Suggest changing "inevitable" to "necessary". 4. Page 2, Line 18: Suggest changing "fitting to the" to "fit the". 5. Page 2, Line 30: Suggest changing "is therefore also conform with" with "and therefore also conforms to the" 6 . Page 3, Line 7: There is a reference here to Table 12 (not Table 1). Is this typical table numbering for GMD, i.e., to consecutively number tables starting with the ones in the Appendix and then continue with tables referred to in the main paper? 7. Page 3, Line 10: Suggest changing "extent" to "extend". 8. Page 4, Line 12: Are root fractions vertically resolved? 9. Page 4, Line 12: Only much later in the paper is it apparent that BAD is not used in the current implementation. It would be useful to note that here. 10. Page 4, Line 24: Do you mean zt $=0$ ? 11. Page 5, Line 30: I don't understand "in case the energy balance for building surface should be solved". Shouldn't it always be solved for? 12. Page 6, Line 3: Are these building types appropriate only for a specific region (e.g., country), or are they generally applicable globally? 13 . Page 6 , Line 4: Suggest changing "build" to "built". 14. Page 6, Line 17: In general, if vegetation is not present, is bare soil parameterized? 15. Page 6, Line 18: Not resolved by the vertical numerical grid? 16. Page 7, Line 10: What types of water surface and parameters are available? Is there a table to be referenced here? 17. Page 10, Line 6: Are the pre-processing scripts referred to here publically available? Or are these simply the same thing as the palm_csd? Are these scripts general enough such that they could be applied to other cites, at least in Germany? 18. Page 11, Line 15: "7, 8"? 19. Page 13: Is there any additional data that is available, now or in the future, that could alleviate this problem (no information on restoration and heat insulation actions)? 20. Page 14: What threshold of NDVI was used? Was it proven to be accurate? Please spell out this first use of the term NDVI (normalized difference vegetation index) (as noted in the next section). 21. Page 15: Please define here what you mean by intensive and extensive

Printer-friendly version

Discussion paper
Interactive comment 
green roofs. Only later on does it become apparent that extensive means trees growing over the roofs. 22. Page 17, Line 4: Units for LAI? $\mathrm{m} 2$ leaf area per $\mathrm{m} 2$ of ground area? Does LAI then vary with time in the model? If so, at what temporal resolution? What part of the model handles this? 23. Page 17, Line 9: What is an "image granule"? 24. Page 17, Line 16: Please spell out IDL. Interactive Data Language? Reference? 25. Page 19, Line 1: It seem like pavement thickness would be required to accurately model heat transfer. Is this considered/available? 26. Page 20: Line 1: How are street type and street crossings used in the model? What is the difference between pavement type and street type? 27. Page 21, Line 3: Change "respcective" to "respective". 28. Page 21, Line 14: Change "in into" to "into". 29. Page 21, Line 27: Change "his" to "their". 30. Tables A1, A3, A5, A6 captions: Please describe each of the symbols given here. In Table A1, what is IFS? In Table A3, what are the units for these parameters? 31. Table A4: Are there values for different depths in the soil? What are the depths? 32. Table A7: By "longwave" and "shortwave" do you mean near-infrared and visible, respectively? 33. Table B2: Is "255" a missing or fill value or something else? 34. Table B3: Is an English translation useful or appropriate here? 35. Table B5: Is buffer width the same as street width? I don't see any cases where "the number of lanes is indicated". Are these in meters? 36. Table B6: Are these in meters? 37. Table B8: Is an English translation useful or appropriate here? 38. Table B9: Is "255 a missing or fill value or something else? 39. Table B11: What is "Biotope"? Change "abbriviation" to "abbreviation". Is an English translation useful or appropriate here? 40. Table 13: This table refers to tables in the supplements, but the only supplement file appears to be the netcdf file that represents the input for the static driver. For example, what is "Tab.S10 in the Supplements"? Please provide more information for readers.

Interactive comment on Geosci. Model Dev. Discuss., https://doi.org/10.5194/gmd-2019-355, 2020.

Printer-friendly version

Discussion paper 